\title{
Tratamiento antitrombótico en pacientes con fibrilación auricular: estudio descriptivo en Atención Primaria
}

L. Carrillo Ramírez, Ma I. Fernández López, M. Sarmiento Gallego,

M $^{a}$ D. García Nadal, J. M. Pérez Fernández, A. Serrano Navarro ${ }^{1}$ Médicos de Familia. 'Enfermero. Centro de Salud de Alcantarilla-Sangonera. Unidad Docente Gerencia de Atención Primaria CAP1. Murcia

\section{RESUMEN}

Fundamento: la fibrilación auricular es la arritmia crónica más frecuente en la población y sus consecuencias, derivadas de su potencial emboligeno, pueden ser graves. Actualmente se recomienda valorar el tratamiento antitrombótico en los pacientes de elevado riesgo de ictus.

Objetivo: conocer el tratamiento antitrombótico de los pacientes con fibrilación auricular en el ámbito de la Atención Primaria, valorando la adecuación del tratamiento a las directrices actuales.

Diseño, material y método: estudio descriptivo transversal. Centro de Salud de Alcantarilla-Sangonera (Murcia); centro urbano de Atención Primaria que tiene adscrita una población de unos 20.000 habitantes. Pacientes: todos los individuos con fibrilación auricular registrados en la base de datos OMI-AP de dicho centro.

Resultados: un total de 124 individuos con fibrilación auricular, el 66,9\% eran mujeres y 33,1\% hombres, con una edad media de 69,77 años. Al menos el 50\% mayores de setenta años. Tienen FA crónica, 95 $(76,6 \%)$. Es habitual no encontrar cardiopatía estructural subyacente $(63,7 \%)$. Es infrecuente encontrar individuos sin factores de riesgo de ictus $(16,1 \%)$, y la casi totalidad $(77,4 \%)$ tienen un riesgo elevado. El 79\% siguen tratamiento antitrombótico (anticoagulantes orales el 50,8\% y antiagregantes plaquetarios el 28,2\%). Se considera que el tratamiento antitrombótico es idóneo en el 70,2\% de los casos. La posibilidad de tratamiento idóneo es significativamente mayor en caso de hombres, menores
Antithrombotic therapy in patients with atrial fibrillation: descriptive study in Primary Health Care

\section{ABSTRACT}

Background: atrial fibrillation is the most frecuent chronic arrithmia in population and it consequences, derived from embolism, may be serious. Actually, to assess the antithrombotic therapy it is advisable in patients with high risk for ictus.

Objective: to review the antithrombotic therapy of patients with atrial fibrillation in the context of Primary Health Care attention, and evaluate the adaptation of such therapy compared with actual guidelines.

Design, material and method: cross sectional study. Centro de Salud Alcantarilla-Sangonera in Murcia (Spain), an urban centre of ambulatory care, which cares are provided to around $20.000 \mathrm{ha}$ bitants. All patients with atrial fibrillation registered at data base OMI-AP were included.

Results: 124 patients with atrial fibrillation were studied, $66.9 \%$ were women and $33.1 \%$ men, the mean age was 69.77 years. At least $50 \%$ were older than seventy. The atrial fibrillation was chronic in $95(76 \%)$ patients. Most of them has no structural cardiac disease $(63.7 \%)$. It is rare to find patients without risk factors for ictus (16.1\%), and most (77.4\%) have a high risk. Seventy-nine per cent are undergoing a antithrombotic therapy (50.8\% oral anticoagulants and $28.2 \%$ antiplatelet therapy). We found the therapy was suitable in $70.2 \%$ of patients. Likelihood of a suitable therapy was higher in men, in younger than 75 years, in patients treated with oral anticoagulants, and with those who are not treated in relation to treated with antiplatelet therapy. 
de 75 años, de pacientes tratados con anticoagulantes y de los que no reciben tratamiento en relación con los tratados con antiagregantes.

Conclusiones: la mayoría de los pacientes con fibrilación auricular, valvular y no valvular, siguen un tratamiento antitrombótico, mayoritariamente anticoagulantes orales, siendo considerada, también en la inmensa mayoría de casos, como idónea la elección de la terapia.

Palabras clave: Fibrilación auricular. Tratamiento antitrombótico. Atención Primaria.
Conclusions: most of patients with atrial fibrillation, valvular or non-valvular, undergo an antithrombotic therapy, mainly oral anticoagulants, and we found therapy as suitable in most of the cases.

Key words: Atrial fibrillation. Antithrombotic therapy. Primary Health Care.

\section{INTRODUCCIÓN}

La fibrilación auricular (FA) es probablemente la arritmia que con más frecuencia requiere, hoy día, atención en el nivel asistencial primario; en los últimos años, además, ha recibido un enfoque terapéutico distinto y esperanzador. La creciente incidencia de la FA en nuestro medio parece estar relacionada con el aumento en la expectativa de vida de la población, así como con el aumento de su detección. Clásicamente se ha considerado la FA como arritmia asociada a valvulopatía reumática, sin embargo, la valvulopatía mitral ha dejado de ser la causa más frecuente de FA, siendo la FA no valvular (FANV) más frecuente en nuestra práctica clínica; hoy día es habitual encontrarla asociada a otras patologías como insuficiencia cardiaca (IC), cardiopatía isquémica $(\mathrm{CI})$ o hipertensión arterial (HTA), aunque existe una proporción no desdeñable de casos de FA aislada, es decir, en la que no existe evidencia de alguna otra patología.

Puede decirse que la FA es la arritmia crónica más frecuente en la población, que es poco común en menores de 50 años y que la prevalencia aumenta con la edad ${ }^{1}$. A partir de varios estudios de base poblacional se estima la prevalencia de la FA en Estados Unidos en un 2,3\% en menores de 40 años, del 5,6\% en mayores de 65 años y sobrepasa el $10 \%$ en mayores de 80 años. Aproximadamente el $70 \%$ de los casos se concentran en el grupo de edad entre los 65 y 80 años; aunque el número absoluto de hombres y mujeres con FA es casi igual, por encima de los 75 años prevalece en mujeres ${ }^{2,3}$.

En España, en un estudio de base poblacional realizado en Talavera de la Reina (Toledo) para conocer la prevalencia de FA en población mayor de 65 años, se encontró una prevalencia de FA del 5,6\%, que aumentaba con la edad, siendo significativa la asociación en el caso de mujeres de más de 80 años $^{4}$.
El principal riesgo de la FA, además de las alteraciones estructurales miocárdicas, estriba en que se asocia a un aumento en la incidencia de embolismos sistémicos, de manera particular de accidentes cerebrovasculares (ACV), así como a una mayor mortalidad. Este riesgo varía ampliamente en función de la edad y de enfermedades coexistentes; así, en el caso de la FANV, la incidencia de ictus en pacientes menores de 60 años puede oscilar entre el 0,5\% anual (similar al de una población control ajustada para edad y sexo) y el 6 ó $7 \%$ anual en pacientes con otras anomalías cardiacas o $\mathrm{HTA}^{3,5-7}$. En pacientes que, además de FA, tienen estenosis mitral reumática, el riesgo de ictus es 17 veces superior en relación con pacientes de edad y sexo comparables. . En lo que se refiere a FA paroxística recurrente, aunque suele aparecer en personas de menor edad, la incidencia de ACV isquémico es similar a la de la FANV crónica, siendo también similares los factores de riesgo ${ }^{8}$.

A pesar de lo descrito, la FA ha sido vista frecuentemente como una patología de poca importancia y es, a menudo, desatendida, probablemente porque en muchos pacientes es poco sintomática. De hecho hasta los años ochenta se consideraba el tratamiento antitrombótico sólo en los casos de FA asociados a valvulopatía reumática ${ }^{1,9}$.

Pretendemos con este trabajo conocer el tratamiento antitrombótico de los pacientes con FA en el ámbito de la Atención Primaria, valorando la adecuación del tratamiento a las directrices actuales.

\section{MATERIAL Y MÉTODO}

Realizamos un estudio descriptivo transversal analizando los pacientes adscritos al Centro de Salud de Alcantarilla-Sangonera (Murcia). Se trata de un centro urbano que atiende a una población de 
unos 20.000 habitantes. La selección de pacientes se realiza en febrero de 2002, utilizando las historias clínicas en soporte informático para la localización de los pacientes con FA. Los códigos CIAP (clasificación internacional en Atención Primaria) que utilizamos para ello fueron los siguientes: K-78 (fibrilación auricular), K-84 (arritmia cardiaca), K-83 (valvulopatía no reumática) y K-71 (valvulopatía reumática).

Incluimos en el estudio a todos los pacientes con FA, crónica o paroxística, aislada o asociada a otras patologías cardiacas.

Consideramos como factores predictores de riesgo tromboembólico (FRT): accidente vascular cerebral transitorio o establecido así como otros embolismos sistémicos previos, la edad superior a 75 años, la presencia de hipertensión arterial, insuficiencia cardiaca clínica o disfunción del ventrículo izquierdo (fracción de eyección inferior al 25\%) e incluimos también, aunque no consideramos como tal factor de riesgo, la presencia de diabetes mellitus 5 .

Definimos diferentes grados de riesgo, así consideramos este como Alto en pacientes con FA y valvulopatía asociada, o edad superior a 75 años o pacientes de cualquier edad con FRT; lo consideramos Medio en pacientes con FA de 65 a 75 años sin FRT y, finalmente, lo consideramos Bajo en pacientes con FA menores de 65 años sin FRT $^{10,11}$.

Establecemos las diferentes indicaciones de tratamiento antitrombótico de la siguiente manera: a) indicación de tratamiento con anticoagulantes (ACO): pacientes con FA asociada a valvulopatía, o pacientes de 65 a 75 años de edad con FA no asociada a valvulopatía (FANV) y FRT, o pacientes con FANV en edades superiores a los 75 años con FRT; b) indicación de tratamiento con ACO o con antiagregantes plaquetarios (AAS): FANV en pacientes menores de 65 años con FRT, o pacientes de edades entre 65 y 75 años con FANV sin FRT, o pacientes mayores de 75 años con FANV sin FRT; y c) ningún tratamiento en pacientes con FANV menores de 65 años y sin FRT. En todos los casos consideramos la FA paroxística recurrente como FA crónica $^{12}$.

A partir de aquí elaboramos unos criterios de idoneidad del tratamiento, en los que distinguimos tres categorías: idóneo, aceptable o inaceptable (Tabla I). En caso de existir contraindicaciones al tratamiento ACO consideramos idóneo el tratamiento con AAS, y la abstención terapéutica en caso de contraindicación del tratamiento con AAS.

Por último, tuvimos en cuenta el nivel de asistencia en el que primordialmente era atendido cada paciente, Atención Primaria o Atención Especializada.

Utilizamos el paquete estadístico SPSS, versión 10.00 para Windows. Los resultados cuantitativos se expresan en valores numéricos o porcentajes y la media con la desviación estándar. Para la asociación de variables se utilizó el test de Chi cuadrado. El límite para la significación estadística se estableció admitiendo un error alfa aleatorio inferior al 5\%.

\section{RESULTADOS}

Se incluyeron en el estudio 124 individuos con FA, de los que $83(66,9 \%)$ eran mujeres y 41 (33,1\%) hombres, con una edad media de 69,77 y una desviación típica de 13,49. La distribución etaria por grupos se muestra en la figura 1. La mayoría de los individuos, $95(76,6 \%)$ tenían una FA crónica, siendo $29(23,4 \%)$ los casos de FA paroxística, y también la mayor parte de ellos, 77 (62,1\%), son controlados principalmente en el nivel de atención especializada, mientras que $45(36,3 \%)$ lo son en Atención Primaria. La presencia de cardiopatía asociada la mostramos en la figura 2, siendo habitual no encontrar cardiopatía estructural subyacente. Los factores de riego para ictus los reflejamos en la figura 3 , y en este caso, lo infrecuente es encon-

\section{Tabla I}

\begin{tabular}{|c|c|c|c|c|}
\hline \multicolumn{5}{|c|}{ CRITERIOS DE IDONEIDAD DEL TRATAMIENTO ANTITROMBÓTICO EN PACIENTES CON FIBRILACIÓN AURICULAR } \\
\hline Edad & Factores riesgo & idóneo & $\begin{array}{l}\text { Idoneidad } \\
\text { aceptable }\end{array}$ & inaceptable \\
\hline \multirow[t]{2}{*}{ Más 75 años } & Sí & $\mathrm{ACO}$ & & No-tratamiento \\
\hline & No & $\mathrm{ACO}$ & AAS & No-tratamiento \\
\hline \multirow[t]{2}{*}{65 a 74 años } & Sí & ACO & AAS & No-tratamiento \\
\hline & No, o DM & ACO/AAS & AAS & No-tratamiento \\
\hline \multirow[t]{2}{*}{ Menos 65 años } & Sí & $\mathrm{ACO}$ & AAS & No-tratamiento \\
\hline & No & AAS/No tto. & No-tratamiento & Tratar con ACO \\
\hline FA+valvulopatía & & $\mathrm{ACO}$ & & No-tratamiento \\
\hline
\end{tabular}

ACO: anticoagulantes orales. AAS: antiagregantes plaquetarios. DM: diabetes mellitus. FA: fibrilación auricular. 


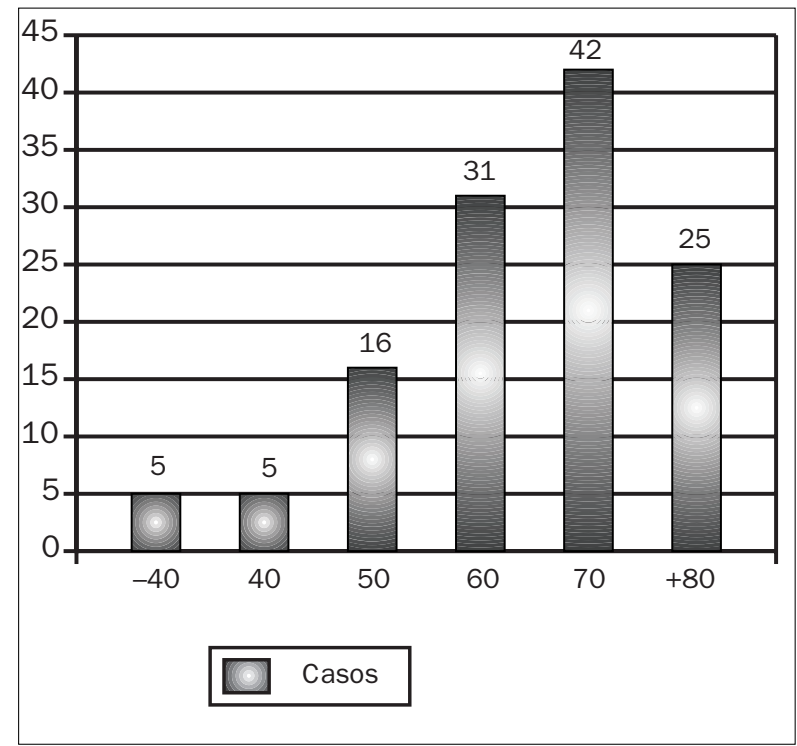

Figura 1

Distribución etaria de pacientes con fibrilación auricular.

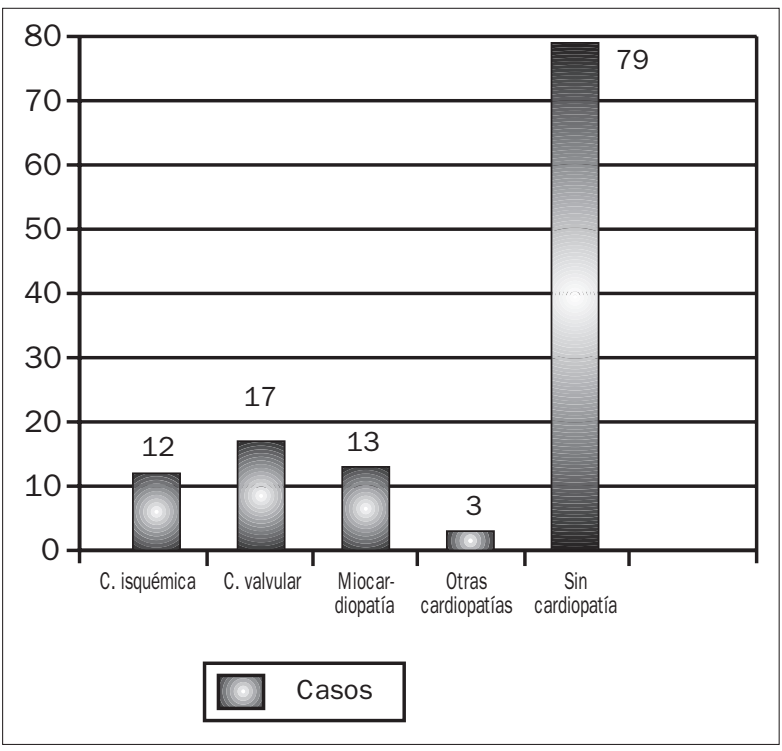

Figura 2

Cardiopatía asociada en pacientes con fibrilación auri-

cular.

trar individuos sin factores de riesgo, de ahí que encontremos que casi la totalidad de los pacientes con FA tienen un riego elevado de ictus, según la presencia de factores de riego, siendo el grado alto en 96 individuos $(77,4 \%)$, medio en $11(8,8 \%)$ y bajo solamente en $17(13,7 \%)$. En las figuras 4 y 5 mostramos, respectivamente, el tratamiento antitrombótico y la idoneidad de dicho tratamiento.

Al realizar la comparación de variables encontramos diferencias significativas en los siguientes

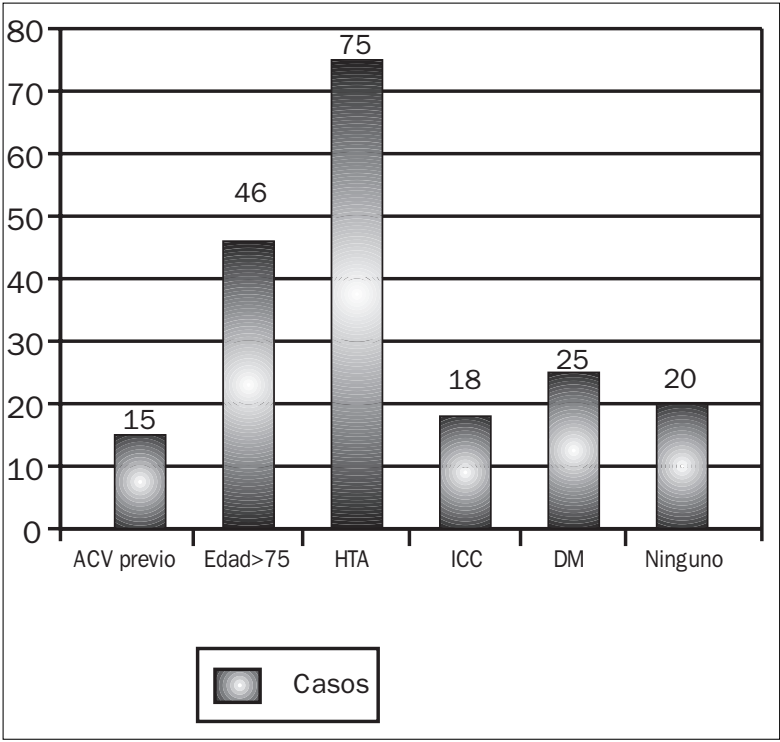

Figura 3

Factores de riesgo de ictus en pacientes con fibrilación auricular. ACV: accidente cerebrovascular; HTA: hipertensión arterial; ICC: insuficiencia cardiaca; DM: diabetes melli-

tus.

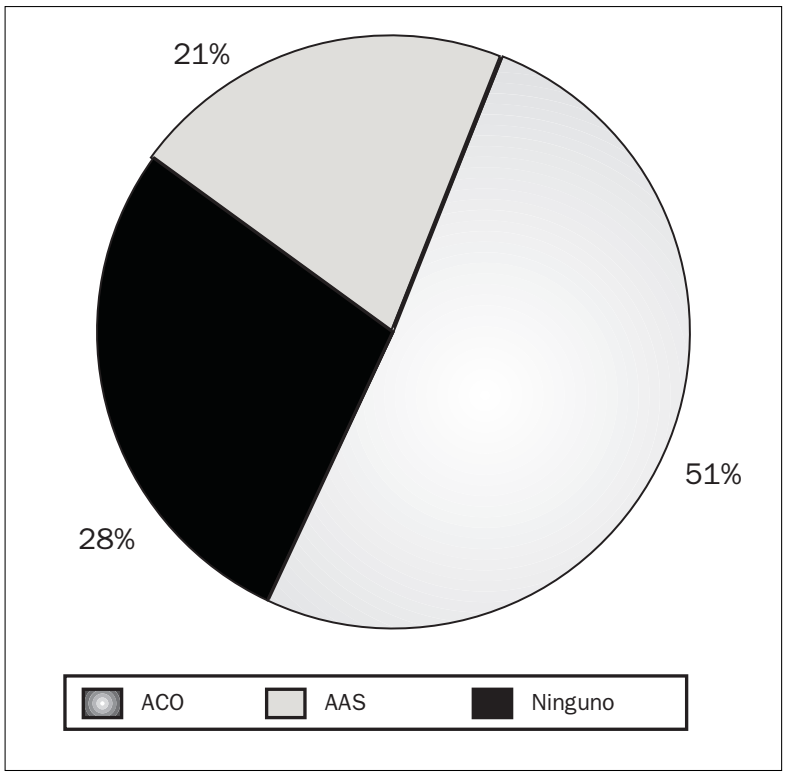

Figura 4

Tratamiento antitrombótico en pacientes con fibrilación auricular. ACO: anticoagulantes orales. AAS: antiagregantes plaquetarios.

casos: a) la posibilidad de estar tratado de manera idónea, frente a inaceptable, era mayor en hombres que en mujeres $(\mathrm{p}<0,01)$ con una odds ratio de 3,77 (IC $1,22 / 12,37)$; b) los individuos menores de 75 años tienen más posibilidades de ser tratados de 


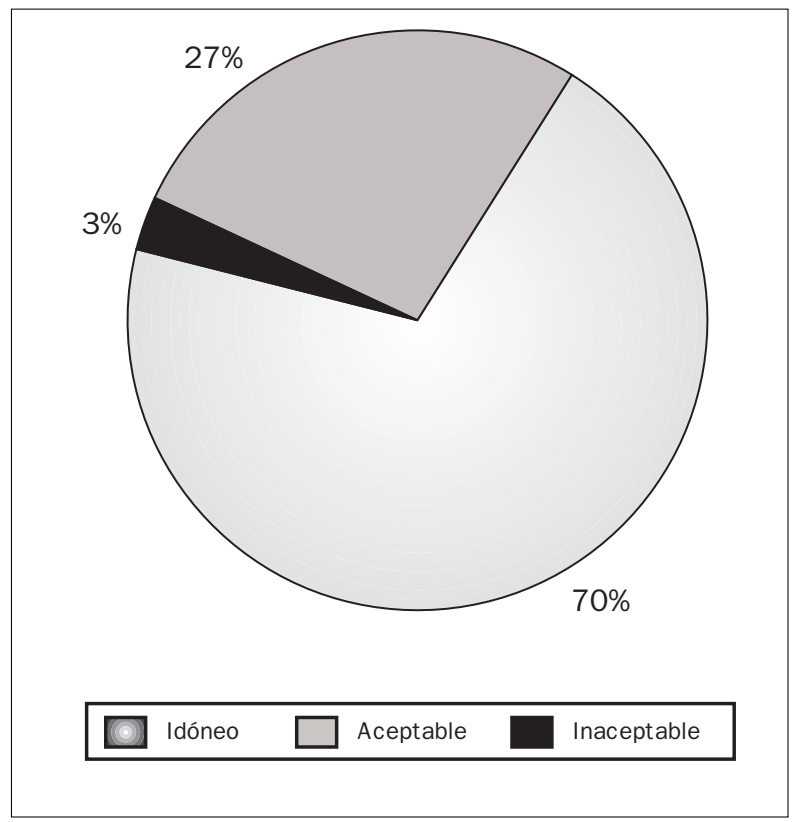

Idoneidad del tratamiento antitromboembótico en pacientes con fibrilación auricular.

forma idónea que los ancianos $(\mathrm{p}<0,001)$, con una odds ratio de 5,57 (IC 2,16/14,58); c) los individuos que no reciben tratamiento con ACO tienen menos posibilidades (odds ratio de 0,07 -IC $0,02 / 0,22-)$ de estar idóneamente tratados $(p<$ 0,001 ); y d) al analizar los tratados con AAS, los que no reciben dicho tratamiento tienen más posibilidades de estar idóneamente tratados (odds ratio $6,26$-IC $2,35 / 16,93-$ y $p<0,001)$. No hemos encontrado diferencias al analizar el comportamiento del resto de variables en relación con la idoneidad del tratamiento antitrombótico.

\section{DISCUSIÓN}

De los pilares básicos en el manejo de la FA, identificar la causa, control de la frecuencia cardiaca, restablecer el ritmo sinusal y prevención de las complicaciones tromboembólicas, éste último es el que, en los últimos años, ha registrado más cambios con repercusión en Atención Primaria, en el sentido de un mayor intervencionismo farmacológico. La clave parece estar en la adecuada selección de pacientes que se beneficiarían hipotéticamente de una terapia antitrombótica. Hemos pretendido conocer la aplicación de estos cambios describiendo lo que acontece en nuestro medio. La baja prevalencia de individuos con FA que obtenemos puede deberse a un infrarregistro de casos, al ser relativamente reciente la implantación del sistema informatizado de historia clínica; esto puede supo- ner un sesgo de selección importante que nos ha sido inevitable asumir. No hemos excluido la FA valvular pues nos parece igual de adecuado valorar su tratamiento; estos casos suponen un 13,07\% del total y cabe destacar la elevada frecuencia de individuos con FA en los que no se encuentra cardiopatía asociada $(63,7 \%)$.

La edad, especialmente mujeres de más de 75 años, la hipertensión arterial, la existencia de ACV previo, de insuficiencia cardiaca o disfunción moderada-severa del ventrículo izquierdo, además de valvulopatía, se consideran en diferentes estudios como factores de riesgo independientes para desarrollar un ictus en pacientes con $\mathrm{FA}^{5,10,13}$. Algunos incluyen la diabetes mellitus ${ }^{3}$ y la dilatación de la aurícula izquierda ${ }^{7}$. En nuestro estudio, solamente el $16,1 \%$ de los casos no tenía ningún factor de riesgo de ictus asociado; la hipertensión arterial $(60,5 \%)$ y la edad superior a 75 años $(37,1 \%)$ fueron los factores de riesgo asociados más frecuentes.

Con fines prácticos, se ha pretendido estratificar el riesgo en diferentes niveles atendiendo a factores clínicos y ecocardiográficos ${ }^{9,11,14}$, aunque el valor de la ecocardiografía es controvertido ${ }^{15}$. En un estudio longitudinal se valoraron tres esquemas para la identificación del riesgo en una cohorte de pacientes con FA sin isquemia cerebral previa en tratamiento con aspirina; los esquemas pudieron identificar los pacientes con bajo riesgo de ACV isquémico, pero menos consistentemente a los de alto riesgo $^{16}$. Nosotros utilizamos sólo criterios clínicos y encontramos que la mayoría de los individuos se sitúan en un nivel de riesgo de ictus alto $(77,4 \%)$ o medio $(8,9 \%)$ susceptibles, por tanto, de tratamiento antitrombótico.

En diversas revisiones que incluyen los grandes estudios prospectivos, aleatorizados para valorar el tratamiento con warfarina o aspirina, solas o en combinación, se verifica la superioridad de la warfarina sobre la aspirina y placebo y, aunque más controvertida, la de aspirina sobre placebo en la prevención primaria del ictus ${ }^{5,7,17-20}$. No obstante, otros autores no encuentran un beneficio tan evidente, en relación con el riesgo, en el tratamiento profiláctico con warfarina en pacientes con $\mathrm{FANV}^{21}$, ni que las diferencias entre warfarina y aspirina sean tan grandes en términos de reducción de la mortalidad cardiovascular a largo plazo ${ }^{22}$. En prevención secundaria, también se ha demostrado la mayor eficacia de la warfarina sobre la aspirina, y de ambas sobre placebo en la prevención de ictus en pacientes con FA no reumática ${ }^{5,23,24}$; aunque también en este caso existen publicaciones que no observaron diferencias en la incidencia de $\mathrm{ACV}^{25}$. Del análisis de los ensayos SPAF (Stroke Prevention in Atrial Fibrillation) I-III se concluye que al menos el $52 \%$ de los ictus en pacientes con FA tienen un origen cardioembólico, y éstos se reducen 
con el tratamiento con warfarina; la aspirina reduciría los ictus no cardioembólicos. Los pacientes de mayor riesgo tienen las mayores incidencias de ictus cardioembólico y tienen la mayor reducción de ictus al tratarse con warfarina ${ }^{26}$.

La mayoría de nuestros pacientes siguen tratamiento antitrombótico, el $51 \%$ con ACO y el $28 \%$ con AAS, datos que son superiores a otros estudios que valoraban el tratamiento en pacientes con FANV en Atención Primaria ${ }^{27-31}$, y son similares a los de otros estudios llevados a cabo en atención especiali$\mathrm{zada}^{32,33}$. Hay que significar que entre los estudios referidos y el nuestro hay al menos cuatro años de separación y es de suponer que sus resultados a día de hoy serían mejores. En nuestro trabajo, la idoneidad del tratamiento antitrombótico es elevada, considerando como idóneos el 70\% y como aceptables el $3 \%$ de las intervenciones, porcentajes que son similares ${ }^{28,29}$ o superiores ${ }^{30}$ a los de estudios precedentes. Coincidimos con otros autores en que las personas con menos edad tienen más posibilidades de ser tratados de manera adecuada que los mayores de 75 años $^{28,29,31}$. En uno de ellos, además, encuentran que el tener realizado un ecocardiograma y los informados del riesgo de tromboembolismo, estarían mejor trata$\operatorname{dos}^{30}$. En este y en otro estudio el tratamiento antitrombótico sería significativamente menor en el nivel de Atención Primaria en relación con el hospitalario $^{30,31}$. Nosotros no hemos encontrado diferencias relacionadas con el nivel en el que son atendidos los individuos, $\mathrm{y}$ sí hay tendencia significativa hacia una mejor adecuación del tratamiento en hombres, aunque puede tratarse de un sesgo al ser este grupo de menor edad media (64,37 años) que el de mujeres (72,43 años). Curiosamente, encontramos que al comparar, en cuanto a idoneidad del tratamiento, el grupo de pacientes tratados con antiagregantes con el que no reciben tratamiento, es más posible que este último sea idóneo, lo que explicamos por la existencia de pacientes que siguen tratamiento con AAS cuando lo idóneo sería estar anticoagulados.
El tratamiento anticoagulante debe monitorizarse cuidadosamente para conseguir un INR entre 2,0 y 3,5 para la mayoría de las indicaciones (a excepción de valvulopatías, que sería mayor); en ancianos puede ser apropiado un límite superior de INR de 3, $0^{13,14,34,35}$. En este trabajo no hemos recogido el grado de control de anticoagulación (entendido como pacientes con INR en rango terapéutico) ni las complicaciones del tratamiento anticoagulante, al haberlo hecho en una reciente publicación ${ }^{36}$. Los riesgos del tratamiento anticoagulante vienen derivados de las complicaciones hemorrágicas severas, cerebrales o gastrointestinales, cuya incidencia oscila entre el 1,3 y el $8,3 \%$ anual dependiendo entre otros factores de la edad del paciente; se registró, además, un $2,8 \%$ anual de recidivas de ictus ${ }^{3,3,7,9,37-39}$.

En conclusión, en nuestro estudio, la mayoría de los pacientes con fibrilación auricular, valvular y no valvular, siguen un tratamiento antitrombótico, mayoritariamente anticoagulantes orales, siendo considerada, también en la inmensa mayoría de casos, como idónea la elección de la terapia. Ello es congruente con estudios previos, mejorando resultados anteriores. En cualquier caso opinamos, como otros autores ${ }^{7,40}$, que a la hora de decidir la indicación de tratamiento preventivo hay que tener en cuenta que la aplicación de protocolos a la práctica clínica dependerá de otros factores como son la edad avanzada, el estado cognitivo, la situación social, posibilidades de seguimiento, existencia o no de cuidadores, tratamiento simultáneos, etc., que son igualmente determinantes en el éxito terapéutico.

\section{CORRESPONDENCIA:}

Luis Carrillo Ramírez

C/ Azalea, 20. Urbanización Ladera

30110 Churra, Murcia

Tfno: 968856006

\section{Bibliografía}

1. Lip G, Beevers D. ABC of Atrial Fibrillation: History, epidemiology, and importance of atrial fibrillation. BMJ 1995; 311: 1361 .

2. Feinberg WM, Blackshear JL, Laupacis A, Kronmal R, Hart RG. Prevalence, age distribution, and gender of patients with atrial fibrillation. Analysis and implications. Arch Intern Med 1995; 155: 469-73.

3. Stern S, Altkorn D, Levinson W. Anticoagulation for chronic atrial fibrillation. JAMA 2000; 283: 2901-3.

4. Labrador MS, Merino R, Jiménez C, García Y, Segura A, Hernández C. Prevalencia de fibrilación auricular en mayores de 65 años de una zona de salud. Aten Primaria 2001;

\section{0: 648-51.}

5. Ezekowitz MD, Levine JA. Preventing stroke in patients with atrial fibrillation. JAMA 1999; 281: 1830-5.

6. Martínez Brotons F, Comité de Redacción de los Protocolos. Protocolos de prevención y tratamiento de la enfermedad tromboembólica (II). Prevención del embolismo de origen cardiaco. Med Clin 1994; 103: 267-70.

7. Codinach Huix P. Fibrilación auricular de causa no valvular: indicaciones de terapia antitrombótica. Med Clin 1996; 106: 586-9.

8. Hart RG, Pearce LA, Rothbart RM, McAnulty JH, Asinger RW, Halperin JL. Stroke with intermittent atrial fibrillation: 
incidence and predictors during aspirin therapy. Stroke Prevention in Atrial Fibrillation Investigators. J Am Coll Cardiol 2000; 35: 183-7.

9. Rodríguez Cumplido D, Casajuana Brunet J, Botinas Martí M. Anticoagulación y fibrilación auricular no reumática. Aten Primaria 1998; 21: 553-5.

10. SPAF Investigators. Patients with nonvalvular atrial fibrillation at low risk of stroke during treatment with aspirin. Stroke Prevention in Atrial Fibrillation III Study. JAMA 1998; 279: 1273-7.

11. Rodríguez Suárez ML, Martínez Trabanco JI, Rodríguez Llorián AC, Cortina Llosa A. Fibrilación auricular en la práctica médica. Aten Primaria 1996; 18: 458-565.

12. Córdoba R, Hernández AC. Fibrilación auricular en el anciano. FMC-Formación Médica Continuada en Atención Primaria 1999; 6: 240-8.

13. Singer DE, Go AS. Antithrombotic therapy in atrial fibrillation. Clin Geriatr Med 2001; 17: 131-47.

14. Matteoli S, Trappolini M, Chillotti FM. Atrial fibrillation and thromboembolic events prevention. State of the art. Minerva Cardioangiol 2001; 49: 1-13.

15. Hart Rg, Halperin JL. Atrial fibrillation and thromboembolism: a decade of progress in stroke prevention. Ann Intern Med 1999; 131: 688-95.

16. Pearce LA, Hart RG, Halperin J. Assessment of three schemes for stratifying risk in patients with nonvalvular atrial fibrillation. Am J Med 2000; 109: 45-51.

17. Hart RG, Benavente O, McBride R, Pearce LA. Antithrombotic therapy to prevent stroke in atrial fibrillation: a metaanalysis. Ann Intern Med 1999: 131: 492-501.

18. The Atrial Fibrillation Investigators. The efficacy of aspirin in patients with atrial fibrillation. Analysis of pooled data from 3 randomized trials. Arch Intern Med 1997; 157: 123740.

19. Segal JB, McNamara RL, Miller MR, Powe NR, Goodman SN, Robinson KA, et al. Anticoagulants or antiplatelet therapy for non-rheumatic atrial fibrillation and flutter (Cochrane Review). The Cochrane Library, Issue 1, 2001.

20. Gullow AL, Koefoed BG, Petersen P, Pedersen TS, Andersen ED, Godtfredsen J, et al. Fixed minidose warfarin and aspirin alone and in combination vs adjusted-dose warfarin for stroke prevention in atrial fibrillation: Second Copenhagen Atrial Fibrillation, Aspirin, and Anticoagulant Study. Arch Intern Med 1998; 158: 1513-21.

21. Green CJ, Hadorn DC, Bassett K, Kazajian A. Anticoagulation in chronic nonvalvular atrial fibrillation: a critical appraisal and meta-analisis. Can J Cardiol 1997; 13: 811-5.

22. Taylor FC, Cohen H, Ebrahim S. Systematyc review of long term anticoagulation or antiplatelet treatment in patients with non-rheumatic atrial fibrillation. BMJ 2001; 322: 3216.

23. Koustaal PJ. Anticoagulants for preventing stroke in patients with nonrheumatic atrial fibrillation and a history of transient isquemic attacks (Cochrane Review). The Cochrane Library, Issue 1, 2001.

24. Hart RG, Benavente O, McBride R, Pearce LA. Antithrombotic therapy to prevent stroke in patients with atrial fibrillation: a meta-analysis. Ann Intern Med 1999: 131: 492501.

25. Yamaguchi T, for Japanese Nonvalvular Atrial Fibrillation-
Embolism Secondary Prevention Cooperative Study Group. Optimal Intensity of warfarin therapy for secondary prevention of stroke in patients with nonvalvular atrial fibrillation. Stroke 2000; 31: 817-25.

26. Hart RG, Pearce LA, Miller VT, Anderson DC, Rothrock JF, Albers GW, et al. Cardioembolic vs. noncardioembolic strokes in atrial fibrillation: frequency and effect of antithrombotic agents in the stroke prevention in atrial fibrillation studies. Cerebrovasc Dis 2000; 10: 39-43.

27. Brotons C, Moral I, Antón JJ, Cobos M, Cucurull E, Gallego $\mathrm{C}$, et al. Tratamiento preventivo de la fibrilación auricular no reumática: de la eficacia de los ensayos clínicos a la efectividad en la práctica clínica. Aten Primaria 1997; 20: 367-71.

28. Galindo G, Peiró R, Plana A, Navarro MA, Berdié J, Morató J. Profilaxis de la enfermedad tromboembólica en enfermos con cardiopatía. Aten Primaria 2000; 26: 293-7.

29. Go AS, Hylek EM, Borowsky LH, Phillips KA, Selby JV, Singer DE. Warfarin use among ambulatory patients with nonvalvular atrial fibrillation: the anticoagulation and risk factors in atrial fibrillation (ATRIA) study. Ann Intern Med 1999; 131: 927-34.

30. Aloy-Duch A, Cuenca-Luque R, Rollán-Serrano E, Casanova-Sandoval J. Utilización de trombolíticos en pacientes con fibrilación auricular crónica en un área sanitaria comarcal. Med Clin 1999; 112: 454-6.

31. Bertomeu V, et al. Estudio CARDIOTENS 99. Empleo de fármacos antitrombóticos en pacientes hipertensos con fibrilación auricular crónica. Estudio CARDIOTENS 99. Med Clin 2002; 118: 327-31.

32. Sánchez JF, García L, Chiquero M, Lozano G, Pérez S, Alonso T, et al. Tratamiento antitrombótico en la fibrilación auricular no reumática. ¿Seguimos las recomendaciones de los ensayos clínicos? An Med Interna 1999: 569-73.

33. Callejas JL, Ortego N, Díaz-Chamorro A, Troncoso E. Utilización de anticoagulación en pacientes con fibrilación auricular crónica no valvular. Med Clin 1999; 113: 679.

34. Hakey GJ. Nonvalvular atrial fibrillation and stroke prevention. National Blood Pressure Advisory Committee for the National Heart Fundation. Med J Aust 2001; 174: 234-9.

35. Koefoed BG, Gullow AL, Petersen P. Prevention of thromboembolic events in atrial fibrillation. Thromb Haemost 1997; 78: 377-81.

36. Sarmiento M, Carrillo L, Fernández Y, Cabrera N. La experiencia de nuestro centro de salud en el tratamiento anticoagulante. Aten Primaria 2001; 28: 138-9.

37. Gullow AL, Koefoed BG, Petersen P. Bleeding complications to long-term oral anticoagulant therapy. J Thromb Thrombolysis 1994; 1: 17-25.

38. Gullow AL, Koefoed BG, Petersen P. Bleeding during warfarin and aspirin therapy in patients with atrial fibrillation: the AFASAK 2 study. Atrial Fibrillation Aspirin and Anticoagulation. Arch Intern Med 1999; 159: 1322-8.

39. Palomeras E, Roquer J, Pou A. Anticoagulación oral en la prevención secundaria de la enfermedad cerebrovascular. Seguimiento a largo plazo de 169 pacientes. Rev Neurol 1998; 27: 772-6.

40. Alonso Roca R, Alvarez Solanes, Alcaraz Betancourt A. Anticoagulación en la fibrilación auricular: implicaciones en atención primaria. Aten Primaria 1998; 21: 500-1. 